\title{
Multimode Pumping of Optical Parametric Oscillators
}

\author{
Larry R. Marshall, Alex Kaz, and Orhan Aytur
}

\begin{abstract}
Calculations suggest that optical parametric oscillators (OPO's) can be efficiently pumped using multimode, divergent pump sources. The influence of pump beam divergence and mode structure upon OPO performance is measured for both noncritical phase-matching, and OPO's with walkoff. Multimode OPO pumping is shown to be efficient, provided appropriate nonlinear crystals and OPO cavities are employed; the nonlinear crystal must have sufficient angular acceptance to tolerate a divergent pump; the OPO cavity must support modes that match the divergence and spatial intensity characteristics of the pump. For low-order pump modes, the OPO can be made to match the mode of the pump. Higher order pump modes reduce the OPO efficiency, and cause a saturation of efficiency with increasing pump power. The efficiency is degraded in a similar fashion in the presence of walkoff. Multimode pumping is more difficult in longer OPO cavities due to increased buildup time of higher order OPO modes.
\end{abstract}

\section{INTRODUCTION}

$\mathbf{O}$ PTICAL parametric oscillators (OPO's) bring wavelength agility to solid-state lasers. Conventional wisdom, and laboratory experience, show that the performance of OPO's is extremely sensitive to the beam quality of the pump source. In addition, the relatively poor quality of available crystals makes optical damage a more frequent occurrence than efficient parametric oscillation. Recent advances in crystal technology have decisively solved the latter impediment. It is now time to address the former.

Poor beam quality affects OPO's in several ways: reduced pump intensity, poor mode matching, divergence exceeding the OPO crystal's acceptance angle, reduced gain guiding, transverse mode competition, broadened OPO linewidth (due to spatially varying phase matching), and increased sensitivity to walkoff. In materials like KTP, with acceptance angles typically greater than $0.4^{\circ}[1]$, focused beams with waists of $100 \mu \mathrm{m}$ can be many times diffraction limited and still remain within the acceptance angle. In an OPO, exceeding the acceptance angle does not preclude parametric oscillation, rather, the signal and idler wavelengths change to phasematch the new propagation angles, thereby broadening the OPO linewidth.

Manuscript received May 17, 1994; revised September 27, 1995.

L. R. Marshall was with Fibertek. Inc 510 Herndon Pky. Herndon. VA 22070 USA. He is now with Light Solutions Corp., Unit 2A3 Concord-Acton Industrial Park, Knox Trail, P.O. Box 2239, Acton, MA 01720-6239 USA

A. Kaz is with Fibertek, Inc., 510 Herndon Pky, Herndon, VA 22070 USA

O. Aytur is with Bilkent University, Department of Electrical and Electronics Engineering, TR-06533 Bilkent, Ankara, Turkey.

Publisher Item Identifier S 0018-9197(96)01110-4.

\section{THEORY}

In general, multimode pumping of KTP OPO's is limited by three factors:

-mode matching of the pump beam to the OPO cavity mode, -buildup time of successive higher order modes, and

-spatial intensity distribution of the pump.

We investigate these factors one by one, making appropriate references to our experiments along the way.

\section{A. Mode Overlap}

The coupling of pump to OPO cavity mode is determined by both the OPO cavity and the beam profile of the pump. Mode matching refers to the manner in which the intensity profile of the pump matches that of the mode defined by the cavity mirrors. In the case of an ideal plane-parallel cavity, the fundamental mode diameter is infinite [2]. In this case, the mode profile (and indeed cavity stability) is controlled by gain guiding [3] due to the transverse intensity profile of the pump. The temporally evolving OPO mode tailors itself to match the transverse intensity profile of the pump. We have experimentally observed that in multimode pumping, energy is first generated in the lowest order transverse mode, then spills out into the higher order modes. (See Section IV.)

One must first determine the threshold for higher order OPO modes. The OPO mode must establish itself before useful energy can be extracted, thus each mode has a threshold in terms of pump intensity and buildup time. We must determine the buildup time of each OPO mode in order to then calculate the conversion efficiency. We can extend the iterative procedure of Byer, which considers the competing effects of diffraction and gain guiding [3], [4], to determine the mode buildup for an $n$ th-order mode pump beam near threshold pump intensity. That iteration can be used here because $n$ thorder beams still propagate according to a function of form $w_{s}(n, z) \approx w_{s}(0)\left(1+\xi(n, z) / w_{s}^{2}(0)\right)$, where $w_{s}(n, z)$ is the spot size of the $n$ th-order signal mode and $\xi(n, z)$ is a binary function describing the mode buildup time (see Section IID). In [1], the size of the polarization driving term was calculated, while here we work with the beam spot sizes which is proportional to the Byer \& Brosnan term. Provided that this multimode beam consists of a superposition of LaguerreGaussian modes, we may use

$$
\left(\frac{\pi}{2 L \lambda_{s}}\right)^{2} w_{s}^{6}+w_{s}^{2}-\frac{w_{p}^{2}}{3.6 \sqrt{n}}=0
$$




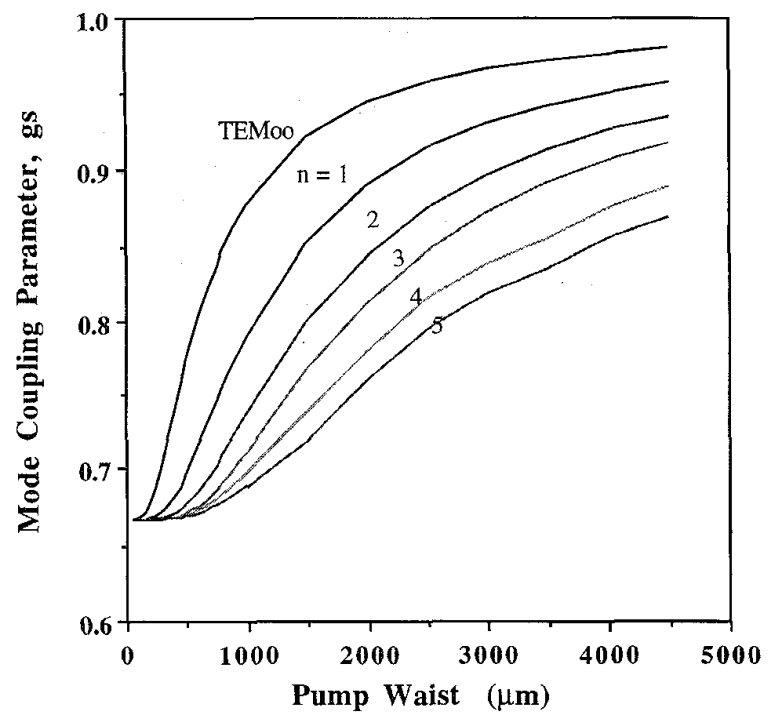

Fig. 1. Calculated effect of pump beam-spot size upon mode coupling parameter, $q_{s}$, for TEM $_{00}$ and multimode pumps (where the number of additional modes " $n$ " is indicated on each curve). The threshold pump intensity calculated for perfect mode-matching is divided by $g_{s}$ to give the increase in threshold caused by mode mismatch.

where $w_{s}$ is the spot size of the signal mode, $w_{p}$ is the spot size of the pump mode, $L(=30 \mathrm{~mm}$ in our experiment $)$ is the OPO cavity length, and $\lambda_{s}(=1570 \mathrm{~nm}$ in our experiment) is the resonated signal wavelength. We use the approximation $w_{n} \approx 1.8 w_{0} \sqrt{n}, n>0$ [2], for the size of the $n$ th-order Gaussian mode. The iteration is achieved using

$$
w_{n, k+1}=w_{n, k} \sqrt{1+\left(\frac{2 L \lambda}{\pi w_{n, k}^{2}}\right)^{2}}
$$

to describe the signal spot size $w_{n, k+1}$ generated on the $(k+1)$ th pass.

In our experiments (detailed in Section III), the TEM $_{00}$ spot size of the pump beam is constant at $w_{p, 0}$, so that the overall beam diameter increases as the mode order is increased. The mode-coupling parameter $g_{s, n}$ is used to determine the threshold of the OPO, by dividing the threshold for perfect mode-matching [4] by $g_{s, n}$, and follows from

$$
g_{s, n}=\frac{w_{p, n}^{2}}{w_{p, n}^{2}+w_{s, n}^{2}} .
$$

The influence of total pump spot size upon mode-coupling parameter, for the $1570 \mathrm{~nm}$ OPO, is plotted in Fig. 1, for $\mathrm{TEM}_{00}$ only and additional higher-order $(n=1$ through 5) pump modes. The mode-coupling parameter increases with $w_{p}$, and the rate of increase is greatest for lower-order modes; thus low-order modes exhibit lower OPO thresholds, as expected. For sufficiently large pump spot sizes, the influence of mode order is reduced, so that large diameter multimode beams should perform almost as well as $\mathrm{TEM}_{00}$ beams, as we have experimentally shown in the past [5], [6]. In the present experiments, the mode overlap improves as the mode order is increased, due to the larger pump diameter.

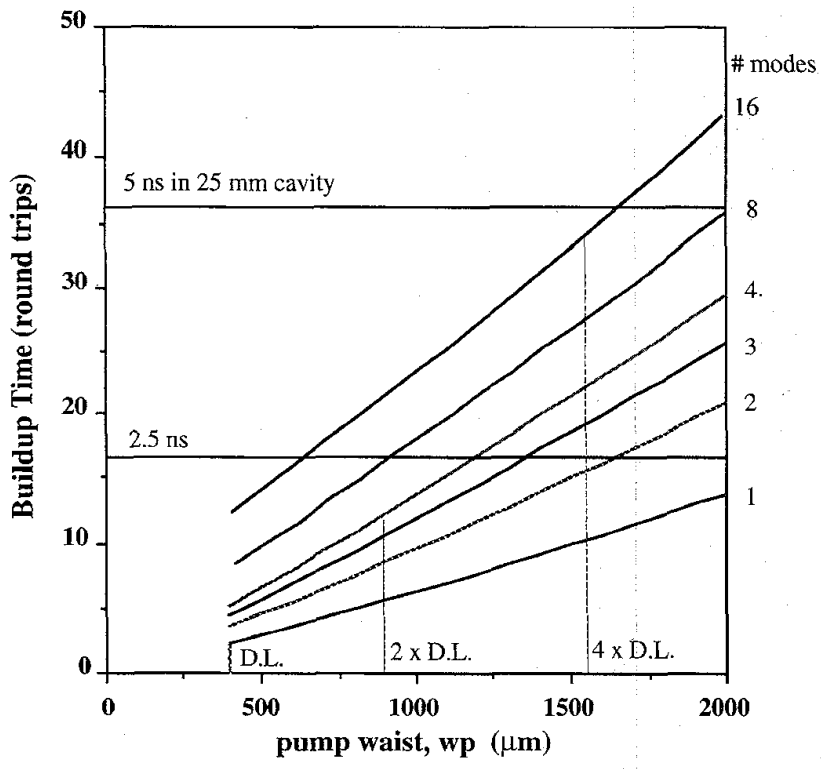

Fig. 2. Calculated buildup time of each OPO cavity mode vs. multimode pump beam spot size. Note that the index of KTP will increase the cavity round trip time, but the pump pulse will also be lengthened in the KTP by the same effect. Therefore, we have ignored this increase for the purposes of comparing the cavity buildup time with the pump pulse length; to determine how much of the pump pulse is capable of contributing to the parametric process. (D.L.: Diffraction Limited.)

\section{B. Temporal Evolution of Cavity Modes}

The temporal dynamics of OPO's are significantly affected by multimode pumping. As with a conventional laser, parametric oscillation builds up from quantum noise over many round trips of the cavity. In multimode pumping, energy must first be generated in the lowest order transverse mode, then spill out into the higher order modes. The mode buildup time is calculated using iterative application of (1) and (2), assuming that each higher order mode originates from the preceding mode. Stable oscillation is achieved when the signal spot size reaches $80 \%$ of its final (steady-state) value. The calculated buildup times are plotted in Fig. 2, for various numbers of pump modes, as a function of pump beam spot size. Our pump pulse duration is $10 \mathrm{~ns}$ in our experiments. (See Sections III and IV for further details.)

According to Fig. 2, the mode buildup time is faster for smaller diameter pumps, and fastest for the fundamental mode. Efficient parametric conversion cannot be achieved until a stable OPO cavity mode is developed. If the pump pulse is too short, a significant amount of the pump energy will be "wasted" before parametric oscillation begins. Experimentally, we have examined the spatial dependence of the OPO output under multimode pumping. Typically, if the OPO cavity is long (>50 mm), one can also observe an increasing delay in the peak of the OPO pulse as the beam is sampled further from the center. For pure TEM $_{00}$ mode pumping, this effect is not observed.

For a $2 \times$ D.L. (two times diffraction limited) pump, the buildup time is less than $2 \mathrm{~ns}$, which is short enough to allow most of the pump pulse to contribute to parametric oscillation. When the beam divergence is increased to $4 \times$ D.L. (i.e., 16 
modes) the buildup time lengthens to $5 \mathrm{~ns}$, which prevents half of the pump pulse from contributing to the OPO efficiency. Thus we expect a significant reduction in OPO efficiency when the pump divergence is increase from 2 to $4 \times$ D.L., from increased buildup time alone.

\section{Pump Beam Uniformity}

The effect of pump beam uniformity involves the ability of the pump to drive the parametric process above threshold over the full aperture of the pump beam. For example, a multimode beam with hot spots may have sufficient intensity to exceed threshold in the hot spots. If these spots are too small, divergence will prevent buildup of a stable OPO cavity mode. It is important to consider the spatial intensity fluctuations of the pump beam in any calculation of OPO efficiency.

\section{Multimode OPO Efficiency}

The OPO efficiency for multimode pumping can be calculated following the work of Bjorkolm [7], and incorporating the above effects. The depleted pump intensity $I_{\text {dep }}(r, t)$ is given by [7], [8]

$$
I_{\mathrm{dep}}(r, t)=I_{0}(r, t) \cos ^{2} \Gamma(r, t),
$$

where $\Gamma(r, t)$ is related to the power gain of the OPO, and defined by the transcendental equation

$$
\sin ^{2} \Gamma(r, t)=\frac{\Gamma^{2}(r, t)}{f(r, t)},
$$

where $f(r, t)$ is the factor by which the pump intensity exceeds threshold for parametric oscillation.

For a Laguerre-Gaussian beam composed of $n$ modes, the factor above threshold $f(r, t)$ varies spatially, with distance $r$ from the center of the beam, and is given by

$$
\begin{aligned}
f(r, t)= & f_{0} g_{s, n}\left(\frac{2}{\pi}\right)^{1 / 4}\left(2^{n} n ! w_{0}\right)^{-1 / 2} H_{n}\left(\frac{r \sqrt{2}}{w_{0}}\right) \\
& \times \exp ^{-2}\left(\frac{r}{w_{0}}\right) \varepsilon_{p}(t)
\end{aligned}
$$

where $H_{n}$ is the $n$ th-order Hermite-Gaussian polynomial [2], $\varepsilon_{p}(t)$, is the normalized temporal envelope of the pump pulse, and $w_{0}$ the spot size of the fundamental mode $(n=0)$. The constant $f_{0}$ is the factor by which the peak of the fundamental mode exceeds threshold; i.e., the peak factor above threshold.

For plane-parallel resonators, the OPO mode is essentially independent of the cavity, and determined primarily by the transverse mode structure of the pump; i.e. by "gain guiding" [3], [4]. This is not the case for OPO cavities with curved mirrors, where care must be taken to overlap the pump beam with the cavity mode, following the required mode size ratio as calculated by Byer [4].

The overall conversion efficiency of the OPO can be obtained by integrating over the spatial extent of the beam, and the temporal evolution of the OPO mode, using

$$
\eta=\int_{0}^{\infty} \xi_{n}(t) d t \int_{0}^{\infty} \frac{\Gamma^{2}(r, t)}{f(r, t)} d r
$$

where $\Gamma(r)$ is defined by (5), and $f(r)$ by (6). The buildup time is included by the binary function $\xi_{n}(t)$ which is unity after the $n$th mode has built up (according to the data of Fig. 2), and zero before that. The solution to (7) is plotted in Fig. 3, as a function of factor above threshold, for the first four modes. While a $\mathrm{TEM}_{00}$ pump reaches $60 \%$ efficiency for a pump intensity five times over threshold, a pump source with four modes, only twice the divergence of the $\mathrm{TEM}_{00}$ beam, converts with only $30 \%$ efficiency. In the high-gain limit, however, the efficiencies all approach a maximum of $60 \%$.

\section{EXPERIMENTAL WORK}

To experimentally verify the above conclusions, we employed a 20 -mm-long, noncritically phase-matched (NCPM) type-II KTP OPO cut for generating a signal wavelength of $1570 \mathrm{~nm}$ when pumped at $1064 \mathrm{~nm}$ [9]. The broad acceptance angle and absence of walkoff, afforded by NCPM, allow us to more closely approximate the ideal conditions assumed in the theoretical analysis above. In addition, we examined a $10 \mathrm{~mm}$ length, $2 \mu \mathrm{m}$ KTP OPO, angle tuned to $\theta=60^{\circ}$ to produce signal and idler wavelengths of 2 and $2.2 \mu \mathrm{m}$. The walkoff for this angle is $\rho=2^{\circ}[1]$. This critically phase-matched crystal, with relatively large walkoff, gives a basis for experimentally determining the influence of walkoff upon efficiency under multimode pupmping.

The pump source was a diode-pumped $\mathrm{Nd}$ : YAG laser, employing back-plane cooled laser-diode arrays capable of operating at up to $5 \%$ duty cycle. These arrays were arranged in circular symmetry, around a 5-mm diameter $\mathrm{Nd}$ : YAG laser rod. By appropriate overlap of the diode outputs in the center of the laser medium, a quasi-Gaussian gain distribution was achieved. When a Gaussian reflectivity mirror [10] was employed to optimize the extraction of $\mathrm{TEM}_{00}$ output power from the pump volume, this device produced $30 \mathrm{~mJ}$ of $\mathrm{TEM}_{00} Q$ switched output in a $10 \mathrm{~ns}$ pulse. The optical cavity consisted of a $-4 \mathrm{~m}$ r.o.c. convex Gaussian reflectivity output coupler with a Gaussian aperture of $1 \mathrm{~mm}$ diameter and $50 \%$ peak reflectivity, and a $2 \mathrm{~m}$ r.o.c. concave back mirror. Within the limits of our measurement capability, the output was diffraction limited (the divergence measured was $1.1 \pm 0.05$ $\times$ D.L.). The same beam quality was obtained using a regular flat output coupler, when a variable intracavity aperture of similar dimensions to the Gaussian aperture, was employed. In this case, the energy was reduced to $15 \mathrm{~mJ}$ in a $10 \mathrm{~ns}$ pulse. The unfocused output was readily doubled with $75 \%$ conversion efficiency using a centimeter cube of KTP cut for type-II doubling at $1064 \mathrm{~nm}$.

The use of a variable intracavity aperture inside the laser cavity allowed the transverse mode quality to be varied in a relatively simple fashion, although this also necessitated adjustment of the $Q$-switch and intracavity polarizer, in order to maintain the same pulse width and envelope. Variations in pulse width and envelope would cause spurious changes in OPO performance.

The unfocused pump beam had a spot size $w_{0}=400$ $\mu \mathrm{m}$, and passed directly into the OPO cavity. We employed 


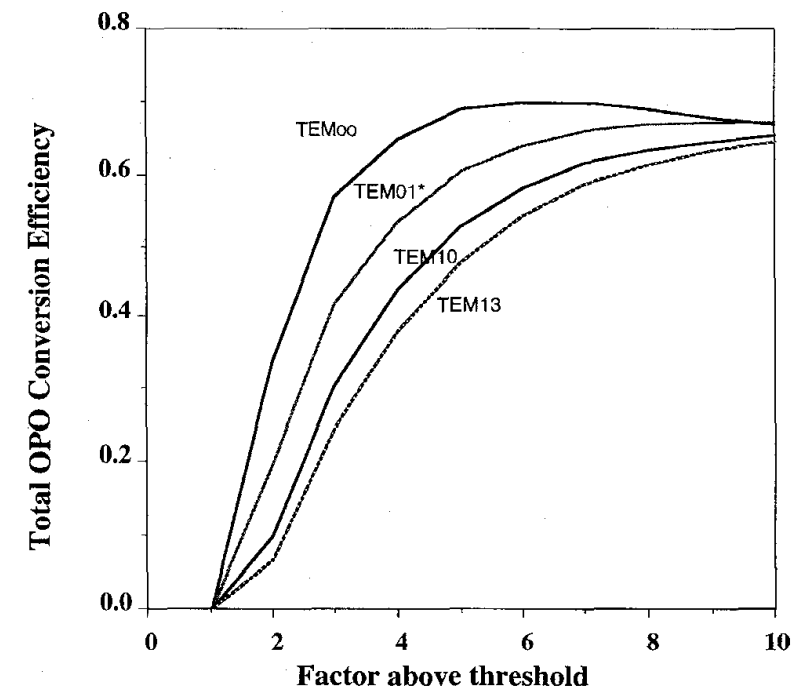

Fig. 3. Calculated total conversion efficiency (to signal and idler) of NCPM OPO, as a function of factor by which pump power exceeds threshold, for various numbers of pump modes.

two types of cavities; flat/flat, and confocal (with a 5 -cm radius of curvature). The former cavity is less sensitive to the effects of mode overlap, as discussed above. The OPO output couplers reflected the pump for a second pass and transmitted $90 \%$ of the resonated signal. The pump and OPO beam divergences were measured simultaneously using two CCD imaging cameras. The laser and OPO outputs were focused to a waist, and the imaging camera used to measure this waist and then measure the beam size at several points in the far field to determine the divergence. The resulting beam profiles are shown in Fig. 4. Note that the imaging capability of the thermal camera used for $1570-\mathrm{nm}$ profiling, is somewhat degraded compared to that used for the 1064-nm pump beam.

The pulse energy for the TEM 00 pump was 4 times above threshold, thus the data of Fig. 4 reflects performance well into the pump depletion regime. As one would expect, the OPO beam profile changes with pump intensity (relative to threshold). Near threshold, only the $\mathrm{TEM}_{00}$ portion of the beam produces OPO output (obviously the higher order modes have higher thresholds). As the pump intensity is increased, the OPO energy spills out into the higher order modes.

\section{RESULTS}

For the diffraction limited pump, the OPO mode is also diffraction limited (with a measured divergence of $1.1 \pm$ 0.1 ). For the three lowest order modes, the OPO produces approximately the same transverse mode in the signal output. As the iris is opened further, and the number of pump modes becomes large, the OPO mode quality degraded. For these highly divergent pumps, the OPO divergence was much greater than the pump, as the OPO cavity mode is determined by the geometrical aperture of the OPO cavity rather than by gain guiding. This is in accordance with (3), above.

We also investigated the influence of pump beam quality upon OPO conversion efficiency. These results are presented

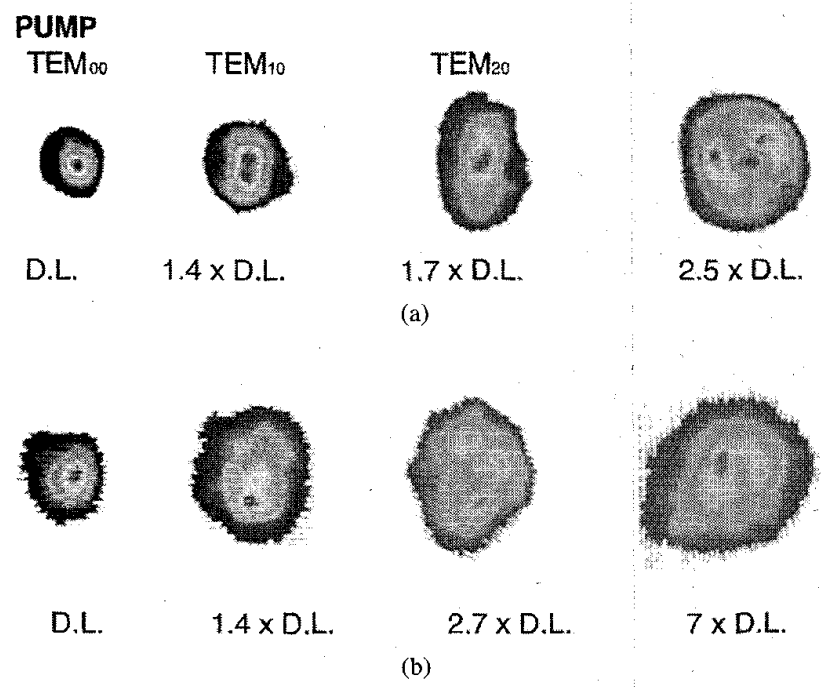

Fig. 4. Measured far field beam profiles of (a) pump, and (b) OPO outputs for various setting of the intracavity iris of the pump laser, when pumping a plane-parallel NCPM KTP OPO.

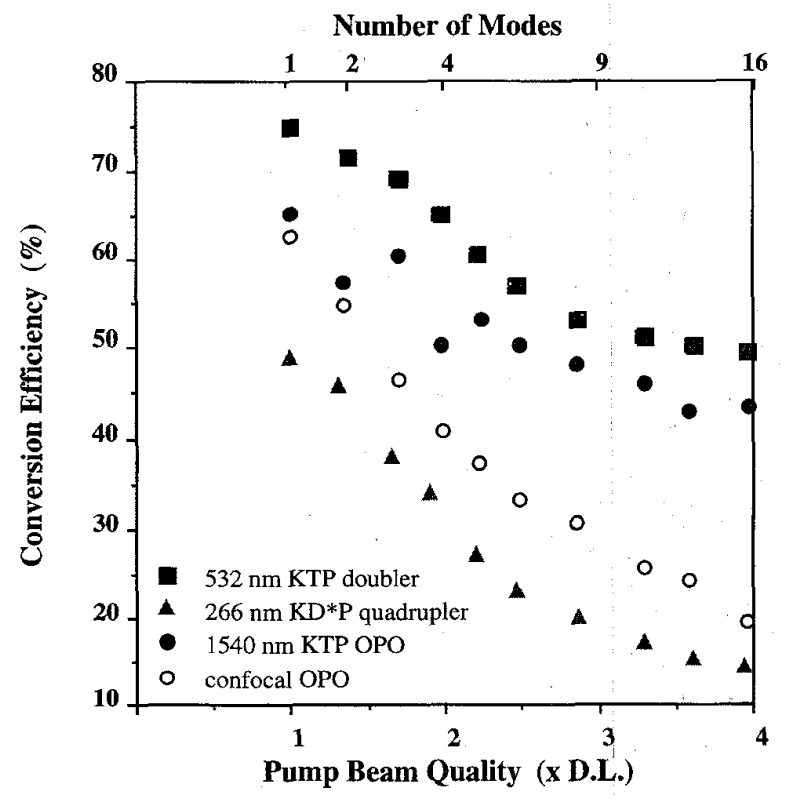

Fig. 5. Conversion efficiency for various nonlinear processes as a function of pump beam divergence. Note that the efficiency shown for OPO's is the total conversion to both signal and idler, and was determined by using the measured signal output to estimate the idler output from photon conservation (in KTP, the idler is largely absorbed).

in Fig. 5. The peak pump intensity for these measurements was kept approximately 4 times greater than the threshold intensity of $50 \mathrm{MW} / \mathrm{cm}^{2}$. From the calculations presented in Fig. 3, we expect that the OPO efficiency will fall from $60 \%$ to $30 \%$ when the pump beam divergence is increased from 1 to 2 times the diffraction limit.

For the low-order modes, the efficiency obtained with a plane-parallel OPO cavity varied with number of modes in a nonuniform manner. Generally, for odd numbers of modes the efficiency was higher, presumably because these modes 


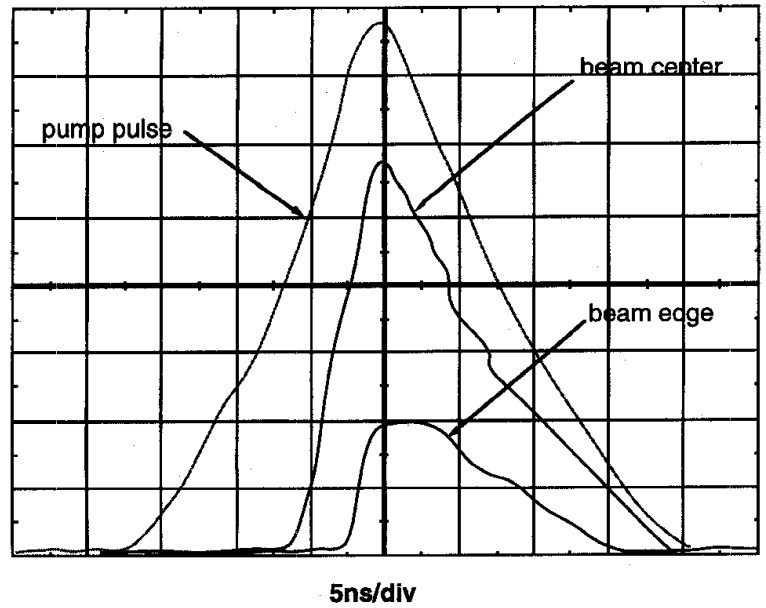

Fig. 6. Scope traces of input pump (faint) and generated signal outputs of short, plane-parallel OPO cavity pumped by $8 \mathrm{~mm}$ diameter $1 \mathrm{~J}$ pump pulse. Traces are shown for far field in the center of the signal output beam, and on the edge.

have higher intensity in the centre of the pump beam. As the number of modes became large, the efficiency decreased steadily, reaching $40 \%$ for a pump divergence of 4 times the diffraction limit. For the plane-parallel OPO, mode-matching appears to be dominated by the pump beam, rather than the OPO cavity, so that the efficiency reduction is caused primarily by the additional buildup time required by higher order OPO modes. As a result, the degradation of efficiency with pump beam quality is not a severe as was calculated assuming effects of both mode-matching and temporal buildup.

Fig. 6 shows two scope traces of signal output taken from our work with large aperture $(8 \mathrm{~mm})$, joule-level pulses [11]. The pump spatial profiles were approximately "top hat" (typical of lamp-pumped oscillator/amplifiers), so that the threshold should be constant across the majority of the beam diameter. Scope traces were taken with a $1 \mu \mathrm{m}$ diameter photodiode placed in the far field. The trace taken near the edge of the OPO output beam shows a lag of about 3 ns compared to the trace taken at beam center. These measurements give an indication that the temporal buildup of the modes within the OPO cavity is similar to that seen with conventional lasers.

The results for the efficiency of the confocal OPO were a little more dramatic than those for the flat/flat cavity. The efficiency was observed to fall rapidly with increased beam divergence. Mode matching appears to dominate the performance of the confocal OPO, and the experimental results compare quite well with the calculations shown in Fig. 3.

To compare other processes and materials, we also measured efficiencies for KTP doubling and $\mathrm{KD}^{*} \mathrm{P}$ quadrupling as a function of beam quality, also shown in Fig. 5. The OPO efficiency degrades more rapidly than the KTP doubling efficiency, but less rapidly than the quadrupling efficiency of KD*P. Since KTP has a large acceptance bandwidth, both the OPO efficiency and the doubling efficiency remain relatively high for divergent pumps. In contrast, $\mathrm{KD}^{*} \mathrm{P}$, with its lower acceptance bandwidth, shows a severe and rapid degradation in efficiency with increasing pump beam divergence.

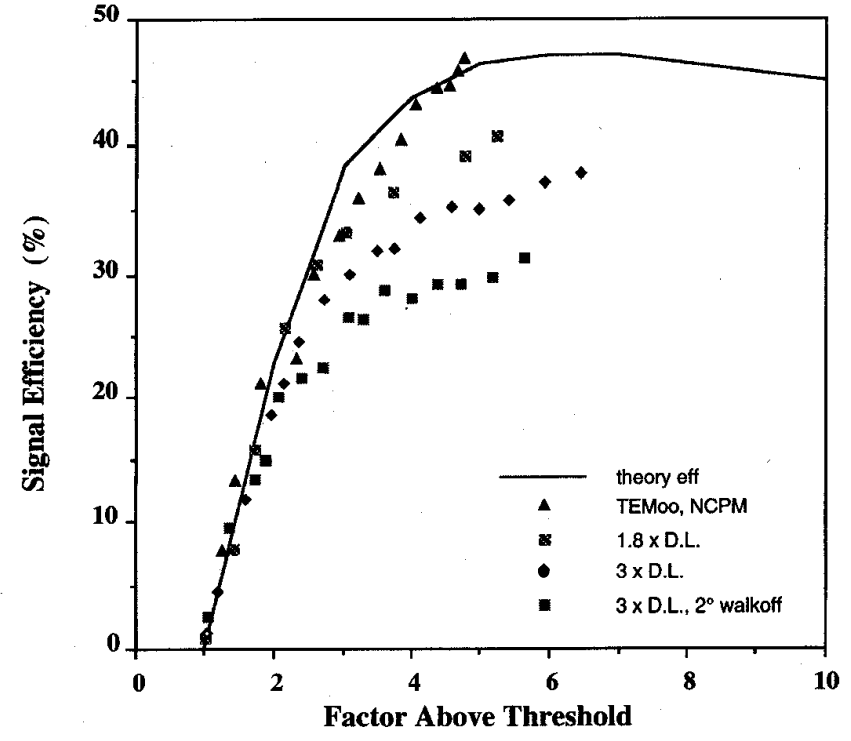

Fig. 7. Measured conversion efficiency (to signal only), as a function of factor above threshold, of noncritically phase matched, and angularly phase matched OPO's, for several pump beam qualities as shown.

\section{DIsCUSSION}

The influence of pump power upon conversion efficiency is shown in Fig. 7, for several pump beam divergences. In multimode pumping, the OPO efficiency increases with pump power above threshold, however, there appears to be a flattening of the efficiency/pump power characteristic, which is predicted by theory. In the presence of walkoff, this flattening is more pronounced as less of the pump beam is able to effectively pump the parametric process.

We also examined the modes produced by the critically phase-matched $2 \mu \mathrm{m}$ KTP OPO with a flat-flat cavity. In the multimode OPO with walkoff, there is a noticeable ellipticity in the output beam in the direction of walkoff. A rough estimate of the effect of this walkoff upon efficiency can be made as follows: as the signal mode "walks off" from the pump mode, the peak of the signal mode sees a pump intensity lower than the peak of the pump. With reference to Fig. 8, the walkoff reduces the maximum available pump intensity to $P_{m}$ from $P_{0}$, so that under optimum conversion $(65 \%)$ the maximum possible OPO intensity is $P_{\mathrm{sm}}$. Walkoff effectively apertures the pump beam, preventing part of the pump crosssection from participating in the nonlinear interaction, as described by Boyd and Kleinman [12].

If the signal is to remain $\mathrm{TEM}_{00}$, then the maximum conversion must still occur at the existing signal peak. Since the available pump intensity is now lower by $\eta_{w}$ than the peak pump intensity, the maximum possible efficiency has also been reduced by $\eta_{w}$. This factor can be approximated by

$$
\eta_{w} \approx \exp \left[-\left(\frac{l \tan \rho}{w_{0}}\right)^{2}\right],
$$

where $l$ is the crystal length. Walkoff is not compounded by the double-pass of the pump, because the pump "walks back on" in the second pass. We note that the Boyd and Kleinman 


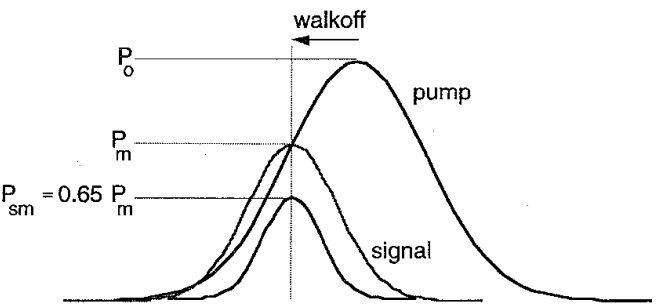

Fig. 8. Idealized conceptual image of walkoff and resulting reduction in OPO efficiency. $P_{0}$ is the actual pump intensity atthe center of the pump beam, $P_{m}$ is the pump intensity experienced by the peak of the signal beam, and $P_{\mathrm{sm}}$ is the peak OPO signal intensity.

theory [12] also predicts an increase in threshold for parametric processes, which would be the inverse of the factor given in (6).

For the $10-\mathrm{mm}$-long $2-\mu \mathrm{m} \mathrm{KTP} \mathrm{OPO}$, we have a walkoff of $\rho=2^{\circ}$, giving a reduction factor of $\eta_{w}=0.9$, for the 1.2-mm multimode pump beam spot size. Experimentally, we observed a reduction in efficiency of about 10 to $15 \%$, which isconsistent with the calculation.

\section{CONCLUSION}

In the absence of cavity mode constraints, the OPO mode structure is determined primarily by the pump beam. For plane-parallel cavities, where mode matching is less critical, the OPO efficiency is reduced by multimode pumping, but remains comparatively high $(45 \%$ for $4 \times$ D.L.). We also calculated that mode matching effects are even less significant when large diameter pump beams are employed. This probably explains the high efficiencies reported in the first multimode, high power OPO experiments we reported in 1991 [5], [6]. However, even when mode matching effects are minimized, there is still a reduction in multimode OPO efficiency caused by increased buildup time. This effect is very noticeable with high energy pumps employing large beam diameters. In other experiments [11], we have seen significant reduction in OPO beam divergence (and energy) simply by lengthening the cavity to suppress higher order OPO modes. Multimode pumping appears worst in confocal cavities which are sensitive to mode matching. In multimode pumping, the OPO efficiency increases with pump power above threshold, however, there appears to be a flattening of the efficiency/pump power characteristic, which is predicted by theory. In the presence of walkoff, this flattening is more pronounced as less of the pump beam is able to effectively pump the parametric process. The future for multimode pumping of OPO's looks quite promising. For energy scaling, larger pump beam diameters will be employed and this will reduce the influence of mode matching (see Fig. 1) upon efficiency. However, the larger diameter pumps will be more susceptible to the increased buildup time required in multimode operation. Short cavity lengths will reduce this effect, but at the same time lead to highly divergent OPO output determined primarily by the geometrical aperture of the pump volume rather than the beam quality of the pump.

\section{REFERENCES}

[1] L. R. Marshall, J. Kasinski and R. L. Burnham, "Diode-pumped eyesafe laser source exceeding $1 \%$ efficiency," Opt. Lett., vol. 16, pp. $1680-1682,1991$

[2] A. Yariv, Quantum Electronics, 2nd ed. New York: Wiley, 1975.

[3] S. J. Brosnan and R. L. Byer, "Optical parametric oscillator threshold and linewidth studies," IEEE J. Quantum Electron., vol. QE-15, pp. 415-443, 1979.

[4] R. L. Byer, in Quantum Electronics, Herbert Rabin and C. L. Tang, Eds. New York: Academic, 1975, vol. I.

[5] R. L. Burnham, L. R. Marshall, J. Kasinski, A. Kaz, and O. Aytur, "Allsolid-state, high power, diode-pumped $455 \mathrm{~nm}$ laser," in Proc. Lasers '91, MD5, Soc. for Optical \& Quantum Electron., 1991.

[6] L. R. Marshall, A. Kaz, and O. Aytur, "New generation diode-pumped tunable lasers," Advanced Solid State Lasers, OSA, vol. 8, pp. 338-342, 1992.

[7] J. E. Bjorkholm, "Some effects of spatially nonuniform pumping in pulsed optical parametric oscillators," IEEE J. Quantum Electron., vol. QE-7, pp. 109-118, 1971.

[8] A. E. Siegman, "Nonlinear optical effects: An optical power limiter," Appl. Opt., vol. 1, no. 6, pp. 739-744, 1962.

[9] L. R. Marshall, A. D. Hays, J. Kasinski, and R. I. Burnham, "An efficient eyesafe source at $1.59 \mu \mathrm{m}$," Advanced Solid State Lasers, OSA, vol. 6, pp. 271-276, 1990.

[10] P. Lavigne, N. McCarthy, A. Parent, and K. Snell, "Laser mode control with variable reflectivity mirrors," Can. J. Phys., vol. 66, pp. 888-895, 1988.

[11] L. Marshall, "Diode pumped lasers: $1 \mathrm{~kW}$ and beyond;" in LASERS '93, Lake Tahoe, NV, Dec. 6-10, 1993, Plenary paper.

[12] G. D. Boyd and D. A. Kleinman, "Parametric interaction of focussed Gaussian light beams," J. Appl. Phys., vol. 39, pp. 3597-3639, 1968.

Larry R. Marshall was born in Sydney, Australia in 1962. He received the Ph.D. degree from the Center for Lasers and Applications.

He joined DOD (Australia) in 1985, and developed novel frequency-shifting and pulse-stacking techniques for high-power lasers, generating efficient blue sources for submarine communication. While with Fibertek since 1990, he developed a new generation of efficient optical parametric oscillators; notably 1.5-1.6 $\mu \mathrm{m}$ eyesafe laser and continuously tunable blue-green sources. He has also developed stable, mode-locked, frequency-doubled, diode-pumped Nd:YAG lasers with multiwatt average powers in the green and IR, for use in the undersea environment. He was Technical Program Manager, responsible for nonlinear optical device and CW-diode-pumped laser research and development. His research interests include nonlinear optics, diodepumped lasers, and oceanography. $\mathrm{He}$ is currently with Light Solutions Corporation.

Alex Kaz, photograph and biography not available at the time of publication.

Orhan Aytur, photograph and biography not available at the time of publication. 